\title{
Memory encoding and hippocampally-based novelty/familiarity discrimination networks
}

\author{
Reza Habib $^{\mathrm{a}, *}$, Anthony R. McIntosh ${ }^{\mathrm{a}}$, Mark A. Wheeler ${ }^{\mathrm{b}}$, Endel Tulving ${ }^{\mathrm{a}}$ \\ ${ }^{a}$ Rotman Research Institute, Baycrest Centre for Geriatric Care, University of Toronto, 3560 Bathurst Street, North York, Toronto, Ont., Canada M6A2E1 \\ ${ }^{\mathrm{b}}$ Department of Psychology, Temple University, Philadelphia, PA, USA
}

\begin{abstract}
Novelty discrimination refers to the ability to decide whether information is new or has been previously encountered. Recent functional neuroimaging work has demonstrated that the hippocampus plays an important function in novelty discrimination. In the study described here, we explored the idea that novelty discrimination does not depend on the hippocampus alone but involves large-scale functional neural networks consisting of spatially remote brain regions. We measured blood flow with positron emission tomography (PET) while subjects semantically encoded visually and auditorily presented situationally novel and familiar words. Following each PET scan, subjects' memory was tested with a standard yes/no recognition test. Blood flow data were analyzed with the covariance-based seed partial least squares (PLS) method. Behaviorally, subjects' recognition performance was higher for novel than familiar words. Neurally, two large-scale functional networks involving the same region of the hippocampus were identified which showed coherent activity either during the encoding of situationally novel (but not familiar) items or situationally familiar (but not novel) items. These findings indicate that different neural networks are active in the processing of situationally novel and familiar information. The observation that the hippocampus participates in both networks supports the principle of neural context.
\end{abstract}

(C) 2002 Elsevier Science Ltd. All rights reserved.

Keywords: Memory encoding; Novelty discrimination; Positron emission tomography; Functional connectivity; Partial least squares

\section{Introduction}

Among the many achievements of organisms is the ability to discriminate between novel and familiar stimuli. Novelty/familiarity discrimination (also known as novelty detection, or novelty assessment) is useful for the organism in a variety of situations, can take a number of specific forms, and can serve several functions (see reviews by [1-4]). The hippocampal system has been found to be critically involved in many tasks involving neural novelty/familiarity (henceforth just 'novelty') discrimination [1,2,5-20]. In this report, we describe a positron emission tomography (PET) study whose purpose was to explore the idea that novelty discrimination does not depend on the hippocampal system alone but is subserved by large-scale neuronal networks which include but go beyond the hippocampal system.

Earlier work suggested that novelty discrimination is a component of the process of encoding incoming information for long-term memory storage [19,20]. Regional cerebral blood flow (rCBF), measured during a yes/no recognition

\footnotetext{
* Corresponding author. Tel.: +1-416-785-2500x3595; fax: +1-416-785-2862.

E-mail address: reza@psych.utoronto.ca (R. Habib).
}

memory test, was higher for novel than for familiar items at a number of sites in the extended limbic system. Other research has corroborated the general thrust of these early findings $[7,9,15,17,18]$, and has extended the novelty assessment networks to other cerebral regions beyond the limbic system, including a number of anterior and posterior neocortical sites $[11,12,19]$. We proposed a specific relation between novelty assessment and memory encoding in the form of a novelty encoding hypothesis. According to this hypothesis, the novelty of incoming information co-determines the extent to which such information is encoded for long-term memory storage: the greater the novelty, the greater the probability of encoding [20]. Since then, the novelty encoding hypothesis has received empirical support from two sources. First, purely behavioral studies have shown that under conditions in which all other possibly relevant variables are held constant, recognition memory is better for novel than familiar information [21,22] (see also [23]). Second, functional neuroimaging studies have identified brain regions whose activity at encoding (i) is correlated with novelty, and (ii) predicts subsequent retrieval of the encoded material [12].

The present study was designed to extend our previous experiment in two ways. First, the novelty/familiarity independent variable has typically been confounded with the task 
outcome: novel test items were (usually) correctly judged to be "new" whereas familiar test items were (usually) correctly judged to be "old". Neuronal differences between the two kinds of material, therefore, could also reflect differences in the task outcome. In the present study, we used a design in which the novelty/familiarity variable was orthogonal to other independent and dependent variables [24], thereby allowing us to examine both behavioral and neural correlates of novelty free of confoudings. Second, the existence of the postulated novelty "networks" was based on indirect evidence, namely observed differences in the activity (blood flow) levels for novel and familiar items in different cerebral regions. In the present study, we sought evidence for functional networks more directly by using the multivariate seed voxel partial least squares method (seed PLS) to identify functional networks of spatially distributed cerebral regions whose activity is correlated across subjects [25-28].

\section{Methods}

\subsection{Subjects}

Eight right-handed male and eight right-handed female subjects between the ages of 18 and $35(\mathrm{u}=24)$ participated in the experiment. Each subject was paid $\$ 80$. The study was approved by the ethics committee of Baycrest Centre for Geriatric Care, University of Toronto.

\subsection{Design}

The experiment consisted of a $2 \times 2 \times 2$ factorial design. Modality of presentation (auditory/visual), levels of processing (semantic/physical), and novelty of material (novel/familiar) were manipulated within subjects. The eight experimental conditions which arise from crossing these three independent variables were counterbalanced across subjects such that each condition appeared in each of the eight possible scan positions. Following each condition, a recognition test was administered. Because of our interest in identifying functional networks underlying the encoding of novel and familiar words into episodic memory, only data from the four semantic encoding conditions, which are more likely to foster long-term encoding, will be reported here $[29,30]$.

\subsection{Material}

A total of 480 five- to eight-letter English words were used (frequency $1-324, \mathrm{y}=25.7$ ). The words were divided into 16 lists of 30 words each. Eight lists were assigned to the auditory condition and the other eight to the visual condition. Within each modality, four lists were assigned to the semantic condition and four lists were assigned to the physical condition. From each set of four lists, one was assigned to the novel condition, another to the familiar condition, and the remaining two served as distractors for the post-scan recognition tests. For the auditory conditions, the words were digitized in a female voice on a personal computer.

\subsection{Procedure}

Prior to scanning, subjects were familiarized with 8 of the 16 word lists (240 stimuli). Four of these lists (120 stimuli) were presented visually and the remaining four (120 stimuli) were presented auditorily. In the first stage of the familiarization phase, subjects were instructed to read or listen to a list of words and try to remember them for a later memory test. Auditory words were presented over speakers connected to a computer. Visual words were presented on a computer monitor suspended over the scanning bed. Words were presented at a rate of $1.5 \mathrm{~s}$ per item. Immediately following the learning phase, subjects were given a recognition test. The recognition test was the second stage of the familiarization phase, and contained only target items from the previous study phase. Subjects, however, were not informed of this fact, and were merely instructed to decide whether they had seen or heard each word. Subjects entered their response by pressing one of two buttons on a computer mouse. The test phase was self-paced. Following familiarization in both modalities, the eight scans, as described earlier, were administered.

Prior to injection of the tracer, subjects were given instructions for the upcoming scan. Depending on the experimental condition, subjects were told that they would either see or hear a list of words, and that they were to either make living/non-living decisions (semantic condition) about each word or decide whether each word began with a vowel (physical condition). No mention of the prior history of the words (novel/familiar) was made. The task was started at the time of radio-tracer injection, which preceded actual blood flow measurement by approximately $30 \mathrm{~s}$. During the task, subjects either heard words spoken over speakers connected to a computer or saw them on a computer screen suspended over the scanning bed. In each scan, one list of words (30 stimuli) was presented at a rate of $3 \mathrm{~s}$ per item plus a $1 \mathrm{~s}$ ISI. Responses were made using a two-button mouse. The task continued for approximately $30 \mathrm{~s}$ after the end of the $1 \mathrm{~min}$ scan.

Immediately after each scan, subjects were administered a recognition test. They were informed that they would either see or hear a list of words and would have to decide whether each word was present in the immediately preceding study list. The test consisted of all of the words presented during the preceding scan and an equal number of distractors. For the novel conditions, the distractors were new words, whereas for the familiar conditions, the distractors were taken from the familiarization phase. Subjects entered their response using a computer mouse. The recognition test was self-paced. This procedure was repeated following each of the eight scans. 


\subsection{Scanning and statistical procedures}

Blood flow was measured with a Scanditronix/GEMS PC 2048-15B PET Scanner using ${ }^{15} \mathrm{O}$-water and $60 \mathrm{~s}$ data acquisition scans [31]. Head movement was minimized with a custom-fitted thermoplastic face mask. Image pre-processing involved realignment of each subject's blood flow images to their first image, spatial transformation into the standard stereotaxic atlas space of Talairach and Tournoux [32], and spatial smoothing using a $10 \mathrm{~mm}$ isotropic Gaussian filter. All pre-processing steps were performed with Statistical Parametric Mapping software (SPM99, Wellcome Department of Cognitive Neurology, London). Prior to the PLS analyses (see below), the data were normalized by setting the mean value of each voxel across the four semantic encoding scans for each subject to 0 .

The main purpose of this study was to identify functional networks [33] of brain structures that participate in the encoding of novel and familiar words. To this end, the data were analyzed with "seed voxel" or seed PLS [25,26,28]. Seed PLS is a multivariate statistical technique that examines the relationship between brain activity (rCBF) in a target region (seed voxel) and brain activity (rCBF) across the whole brain as a function of the different experimental conditions. The seed PLS procedure consists of three steps. (1) The computation of the correlation coefficient between brain activity in the seed voxel and brain activity at every other voxel in the brain across subjects and within conditions. This procedure produces one correlation map per experimental condition. (2) The correlation maps from the different experimental conditions are combined into a matrix and decomposed with singular value decomposition (SVD). SVD produces mutually orthogonal variables (latent variables; LVs), each consisting of a singular image, a singular profile, and a singular value. The singular image identifies patterns of brain regions whose activity covaries, as a whole, with activity in the seed voxel. Each voxel in the singular image is assigned a salience value, a number representing how strongly the voxel represents the experimental effect expressed in the singular profile. A large positive salience means that the voxel strongly expresses the effect, whereas a strong negative salience means that the voxel strongly expresses the opposite of the effect. The singular profile indicates the nature of the relationship between activity in the seed voxel and activity in the regions identified in the singular image as a function of the different experimental conditions. The singular value is a measure of the covariance between the singular image and the singular profile and indexes the percentage of overall covariance accounted for by each latent variable. The latent variables are generated in decreasing order of the singular value, thus the first LV accounts for the largest proportion of overall covariance and the last LV accounts for the least. (3) Multiplication of the singular image by the raw rCBF images (dot product) for each subject results in individual brain scores. The brain score is an indication of how much of the pattern represented in a singular image is expressed by a subject within a condition and is conceptually similar to a factor score from factor analysis. The correlation between blood flow in the seed voxel and the brain scores across subjects and within conditions results in scan profiles which are proportional to the singular profiles but have a simpler interpretation because they are correlations. If these correlations differ between experimental conditions, then the singular image will have identified those brain regions whose functional connectivity with the seed voxel varies as a function of the experimental manipulation.

For each latent variable, the reliability of the identified brain regions in the singular image was assessed by means of a bootstrap estimation of the standard error. Bootstrapping assesses the reliability of activations by guarding against the effects of outlier observations. In bootstrapping, subjects are randomly selected into the analysis with replacement from the entire group of subjects. For each new sample, the entire PLS analysis is re-calculated. This sampling and analysis procedure was carried out 100 times, resulting in estimates of the standard error of the salience at each voxel. The ratio of the saliences to their standard errors is similar to a $z$-score. We considered clusters of 15 or more voxels in which this ratio was greater than $3.29(P<0.001$ on a two-tailed normal distribution) to represent reliable voxels. Orthogonal Procrustes rotation was used to correct for arbitrary axis reflection and rotation during resampling [34].

In addition to assessing the reliability of the voxels in each singular image, the significance of each latent variable was assessed by a permutation test. In this procedure, each subject's data were randomly reassigned without replacement to different experimental conditions, and the entire PLS procedure repeated. Following 500 such randomizations, the number of times the singular value from the randomized PLS analysis exceeded the singular value from the original PLS analysis was noted.

\section{Results}

\subsection{Behavior}

Post-scan recognition scores (hits, false alarms, and corrected recognition) for each condition are shown in Table 1. The corrected recognition data were submitted to a $2 \times 2$

Table 1

Post-scan recognition performance

\begin{tabular}{lcc}
\hline & Visual & Auditory \\
\hline Novel & & \\
Hits & 0.91 & 0.90 \\
FA & 0.04 & 0.03 \\
Corrected & 0.87 & 0.87 \\
Familiar & & \\
Hits & 0.85 & 0.90 \\
FA & 0.07 & 0.09 \\
Corrected & 0.78 & 0.81 \\
\hline
\end{tabular}


(modality $\times$ novelty) repeated-measures analysis of variance (ANOVA). The ANOVA revealed a significant main effect of novelty, $F(1,15)=13.47, P<0.01$, MSe $=5.21$, with recognition of novel words $(0.87)$ being higher than that of familiar words (0.80). Neither the main effect of modality nor the modality by novelty interaction were significant, both $F<1$.

\subsection{Blood flow}

For seed PLS analysis, the first step is the selection of the seed voxel. Typically, this voxel is chosen based on previous analyses of the data, theoretical motivation, or both. In the present case, we targeted the hippocampus proper for the selection of the seed voxel because previous research has demonstrated the involvement of the hippocampus in novelty assessment $[7,11,12,17-20]$. Because of the size of the hippocampal region and evidence for its functional heterogeneity $[17,35,36]$, however, the selection of a single representative voxel (a single coordinate in Talairach space) can be difficult. We proceeded by selecting a single voxel in each hippocampal slice along its long axis. The Talairach Daemon (TD) database $[37,38]$ defines the hippocampus along seven contiguous slices at $4 \mathrm{~mm}$ intervals $(Z=-20,-16$, $-12,-8,-4,0,4)$. Within each slice, we took a voxel at the center of that slice in the $X$ - and $Y$-dimensions (the mean coordinate in both $X$ - and $Y$-dimensions). Because of the size of spatial autocorrelation in PET ( $\sim 10 \mathrm{~mm}$ [39]), the center of each hippocampal slice can be considered to be representative of activation within the entire slice. The seven coordinates that were examined were: (1) $-27-10$ -20 , (2) $-30-14-16$, (3) $-30-18-12$, (4) $-30-24$ $-8,(5)-30-33-4,(6)-29-380,(7)-28-394$. The right hemisphere homologue of these seven coordinates were also examined. For each coordinate, a seed PLS analysis as described above was performed with the goal of identifying a network of brain regions, involving the hippocampus, whose activity distinguishes encoding of novel words from the encoding of familiar words.

We observed two voxels in the ventral hippocampus, -30 $-14-16$ and $-30-18-12$, that identified patterns of brain regions whose collective activity correlated with the activity of these seed voxels differentially as a function of the encoding of novel and familiar words. Because of their proximity (less than $6 \mathrm{~mm}$ apart), these voxels are likely to be part of a single larger cluster rather than representing functionally heterogeneous regions within the hippocampus. Therefore, we chose to run a single seed PLS analysis with both voxels included as seeds. The combined seed PLS analysis identified two significant latent variables. The first latent variable, accounting for $31 \%$ of the overall covariance $(P<0.001)$, identified a pattern of brain regions whose activity correlated with activity in the seed voxels when subjects encoded novel words but not when they encoded familiar words (see Fig. 1 left column middle row). The correlation coefficients were significantly $(P<0.05)$ greater than 0 in the two novel encoding conditions (visual $r=0.81 / 0.81$, auditory $r=0.88 / 0.92$; first seed/second seed) but not in the two familiar encoding conditions (visual $r=0.02 /-0.16$, auditory $r=-0.32 /-0.50$ ). The singular image depicting this pattern is shown at the top left of Fig. 1 (the seed voxel is circled in black). Brain regions whose activity correlated positively with activity in the seed voxel (positive saliences) are shown in yellows and reds, whereas brain regions whose activity correlated negatively with activity in the seed voxel are shown in blues. Coordinates for both sets of regions are listed in Table 2. The interregional correlations amongst this set of regions, coded in terms of colors, are shown at the bottom left of Fig. 1. Each row/column of the correlation matrices represents a single voxel in the singular image. The first two columns, separated from the rest of the matrix, represent correlations with the seed voxels. The order of these voxels match the list of coordinates presented in Table 2. The correlation matrices show the correlation between activity at a

Fig. 1. This figure identifies two distinct patterns of brain regions whose activity is intercorrelated when subjects encode novel words but not familiar words (LV1; left column) or familiar words but not novel words (LV2; right column). The top row identifies the brain regions associated with each pattern, overlayed on an average MRI image $(z=-28$ to 36 by $8 \mathrm{~mm}$ increments). These patterns were identified with a single seed PLS analysis; the seed region (left ventral hippocampus; $-30-14-16$ and $-30-18-12$ ) is circled in black. Regions whose activity correlated positively (positive saliences) with activity in the seed region are shown in yellow, whereas regions whose activity correlated negatively (negative saliences) with activity in the seed region are shown in blue. Coordinates for these regions are listed in Table 2 . The second row of the figure displays the scan profiles as scatter plots and linear regression lines, demonstrating the relationship between standardized activity (rCBF) in the seed voxels (ordinate; red markers $=-30$ $-14-16$, yellow markers $=-30-18-12$ ) and standardized activity (brain scores) across the entire pattern of brain regions (abscissa) in each of the four experimental conditions. The scatter plots on the left show that activity in the two seed voxels is related to activity in the entire pattern only during the novel encoding conditions. The scatter plots on the right show that activity in the two seed voxels is related to activity in the entire pattern during the familiar encoding conditions and the auditory novel encoding condition, but not the visual novel encoding condition. The final row of the figure shows the correlation between activity in the brain regions identified in each pattern across the four experimental conditions. Each row/column in each correlation matrix represents one voxel from the corresponding pattern. The order of the columns correspond to the order of the coordinates listed in Table 2. Positive correlations between brain regions are indicated in yellows and reds, whereas negative correlations are indicated in light and dark blues (the correlation matrix is symmetrical about its main diagonal, colored dark red). The first two columns (separated from the rest of the matrix) show the correlation between activity in the seed voxels (first column $=-30-14-16$, second column $=-30-18-12$ ) and activity in the other regions of the pattern. The four correlation matrices on the left show that the brain regions identified in LV1 are intercorrelated when subjects encoded novel but not familiar words, whereas the correlation matrices on the right show that the brain regions identified in LV2 are intercorrelated when subjects encode familiar but not novel words. 


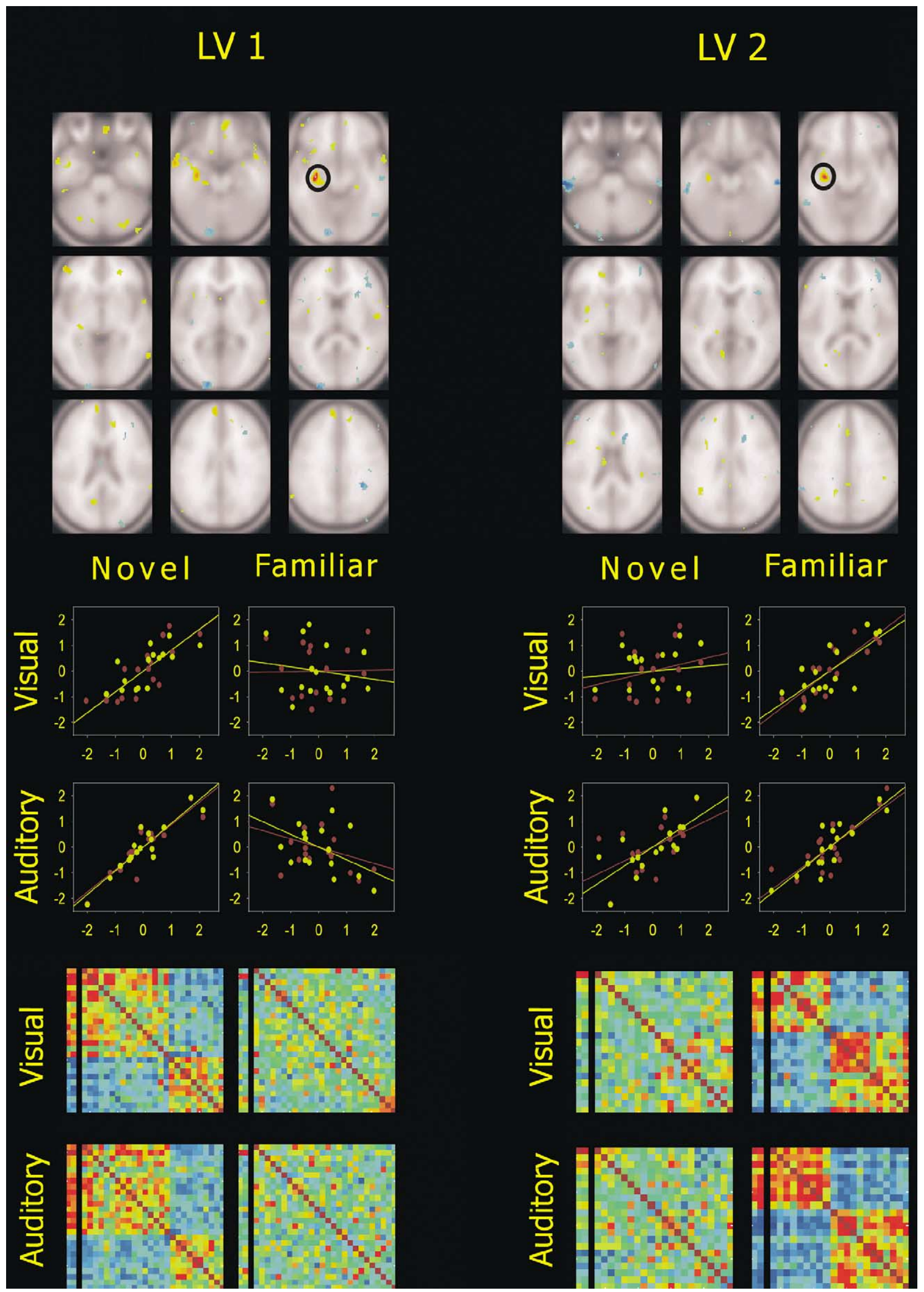


Table 2

Coordinates of brain regions, identified by seed voxel PLS, whose activity is intercorrelated with activity in the hippocampus differentially during the encoding of Novel and Familiar words

\begin{tabular}{rrrl}
\hline$X$ & $Y$ & $Z$ & \multicolumn{2}{l}{ Brain region } \\
\hline \multicolumn{3}{l}{ Latent variable 1-positive saliences } \\
34 & -74 & -28 & Cerebellum \\
-30 & -16 & -16 & Hippocampus \\
4 & 48 & -28 & Orbital Gyrus (BA 11) \\
-28 & -68 & -24 & Cerebellum \\
46 & 10 & -20 & Superior Temporal Gyrus (BA 38) \\
-6 & 24 & -12 & Medial Frontal Gyrus (BA 11) \\
54 & 8 & 8 & Precentral Gyrus (BA 44) \\
-32 & 20 & -8 & Inferior Frontal Gyrus (BA 47) \\
-48 & 52 & -8 & Middle Frontal Gyrus (BA 47 / 10) \\
24 & 44 & -4 & Middle Frontal Gyrus (BA 11) \\
62 & -66 & 0 & Inferior Temporal Gyrus (BA 37) \\
-8 & -64 & 16 & Posterior Cingulate (BA 31) \\
52 & -10 & 16 & Postcentral Gyrus (BA 43) \\
-8 & 52 & 32 & Superior Frontal Gyrus (BA 9) \\
14 & 34 & 24 & Anterior Cingulate (BA 32) \\
-36 & 32 & 40 & Middle Frontal Gyrus (BA 8)
\end{tabular}

Latent variable 1 -negative saliences

$\begin{array}{rrrl}-14 & -86 & -16 & \text { Lingual Gyrus (BA 18) } \\ 52 & -66 & -12 & \text { Fusiform Gyrus (BA 19) } \\ 58 & -18 & -12 & \text { Middle Temporal Gyrus (BA 21) } \\ -24 & -102 & 12 & \text { Middle Occipital Gyrus (BA 18) } \\ 30 & 24 & 20 & \text { Inferior Frontal Gyrus (BA 45) } \\ 40 & 32 & 12 & \text { Middle Frontal Gyrus (BA 46) } \\ 28 & -92 & 16 & \text { Middle Occipital Gyrus (BA 19) } \\ 30 & -44 & 36 & \text { Inferior Parietal Lobule (BA 40) } \\ 44 & -22 & 44 & \text { Postcentral Gyrus (BA 2) } \\ 52 & 12 & 44 & \text { Middle Frontal Gyrus (BA 6 / 8) }\end{array}$

Latent variable 2-positive saliences

$\begin{array}{rrrl}-30 & -16 & -16 & \text { Hippocampus } \\ -8 & 44 & -4 & \text { Anterior Cingulate (BA 32) } \\ -10 & -58 & 4 & \text { Lingual Gyrus (BA 18) } \\ -10 & -16 & 20 & \text { Thalamus } \\ -4 & -40 & 32 & \text { Cingulate Gyrus (BA 31) } \\ -38 & -48 & 28 & \text { Superior Temporal Gyrus (BA 39) } \\ -18 & -52 & 32 & \text { Cingulate Gyrus (BA 31) } \\ -10 & 32 & 44 & \text { Medial Frontal Gyrus (BA 8) } \\ -50 & -16 & 40 & \text { Postcentral Gyrus (BA 3) }\end{array}$

Latent variable 2 -negative saliences

\begin{tabular}{rrrl}
-16 & -92 & -28 & Cerebellum \\
-58 & -26 & -28 & Fusiform Gyrus (BA 20) \\
62 & -24 & -24 & Inferior Temporal Gyrus (BA 20) \\
-56 & -48 & -8 & Middle Temporal Gyrus (BA 37) \\
40 & 48 & -8 & Middle Frontal Gyrus (BA 11) \\
38 & 22 & 4 & Inferior Frontal Gyrus (BA 45) \\
42 & 38 & 12 & Inferior Frontal Gyrus (BA 46) \\
-32 & 40 & 12 & Middle Frontal Gyrus (BA 10) \\
-44 & 44 & 20 & Middle Frontal Gyrus (BA 10) \\
16 & 16 & 24 & Cingulate Gyrus (BA 32) \\
-25 & 46 & 32 & Superior Frontal Gyrus (BA 9) \\
-60 & -46 & 40 & Inferior Parietal Lobule (BA 40) \\
\hline
\end{tabular}

given voxel (or seed voxel) and activity at every other voxel in the singular image. These matrices indicate that during the encoding of novel but not familiar words, the activity of regions with positive saliences (regions colored yellow on the singular image) correlated positively with each other (red square at top left of the Novel-Visual and Novel-Auditory correlation matrices), the activity of regions with negative saliences (regions colored blue on the singular image) also correlated positively with each other (red square at the bottom right of the Novel-Visual and Novel-Auditory correlation matrices), but the activity of these two sets of regions correlated negatively with each other (blue square at the bottom left/top right of the Novel-Visual and Novel-Auditory correlation matrices). The difference in correlation strength between the Novel and Familiar conditions (across all voxels) was tested by means of a permutation test. The results revealed that the interregional correlations were collectively significantly higher (both positively and negatively) during the Novel encoding condition than the Familiar encoding condition (visual $P<0.005$, auditory $P<0.001$ ).

The second latent variable, accounting for $25 \%$ of the overall covariance $(P<0.05)$, identified a pattern of brain regions whose activity correlated with activity in the seed voxels when subjects encoded familiar words and auditorily presented novel words but not when they encoded visually presented novel words (see Fig. 1 right column middle row). The correlation coefficients were significantly $(P<0.05)$ greater than 0 in the two familiar encoding conditions (visual $r=0.84 / 0.74$, auditory $r=0.80 / 0.87)$ and the auditorily presented novel condition $(r=0.53 / 0.73)$ but not in the visually presented novel encoding condition $(r=0.26 / 0.10)$. The singular image depicting this pattern is shown at the top right of Fig. 1 and the coordinates of its component regions are listed in Table 2. The interregional correlations for this set of regions are shown at the bottom right of Fig. 1. The correlation matrices indicate that during the encoding of familiar but not novel words, the activity of regions with positive saliences correlated positively with each other, the activity of regions with negative saliences also correlated positively with each other, but the activity of these two sets of regions correlated negatively with each other. The overall interregional correlation strengths in the Familiar condition were significantly stronger than in the Novel condition (visual $P<0.005$, auditory $P<0.005$ ).

\section{Discussion}

The purpose of the present paper was to explore the idea that novelty discrimination in the brain is subserved by large-scale functional neuronal networks. A network here is defined as a pattern of spatially remote brain regions whose activity levels (rCBF) are correlated (coherent), or functionally connected [33], across subjects regardless of whether the average level of activity in any single component of the network is different between the experimental conditions. To examine this question, we measured rCBF, with PET, while subjects made semantic judgments about visually and auditorily presented lists of common words that were either situationally novel (encountered for the first time at encoding) or situationally familiar (encountered twice before 
earlier in the experiment). Following each PET scan, we also measured subjects' recognition memory for such semantically processed and incidentally encoded words. In order to identify networks underlying novelty discrimination, we used the covariance-based seed PLS data analysis method $[25,26,40,41]$.

The central finding of this study was that large-scale neural networks do exist that distinguish between the encoding of situationally novel and situationally familiar items. It is important to remember that a network is defined in terms of the coherent activity of its components, regardless of whether or not the overall level of activity of these component regions distinguishes between the novel and familiar encoding conditions. Under the conditions of our study, two such networks were found. Each network consisted of two subsets of regions: a set whose activity correlated positively with the activity in the seed voxel and with each other, and another set whose activity correlated negatively with the activity in the seed voxel but positively with each other. The activity of these two sets of regions was negatively correlated with each other. Additionally, by design, each network was common to both visual and auditory modalities $[11,39]$.

The two networks differed in that one was coherent for novel items but not familiar items, whereas the other was coherent for familiar items but not novel items. For convenience, we refer to the first as a novelty network and the second as a familiarity network. It is important to note that it is the selectivity of the network for one kind of material but not the other that makes them interesting and distinguishes them from other types of networks that have been previously discussed in the literature [28,33,42-46].

Our work was initially motivated by an interest in the role that novelty detection may play in long-term memory encoding, along the lines suggested by the novelty encoding hypothesis [19]. In this respect, we wish to note that the behavioral data of our study were clear in showing that, in keeping with the novelty encoding hypothesis and previous findings [21-23], recognition of novel words was higher than that of familiar words. This outcome occurred under conditions where all other experimental conditions, and especially the lexical and semantic identity of the words, was held constant. Therefore, we can conclude that the novelty and familiarity network described here differentiate not just between verbal information previously encountered in the experiment and that not encountered, but between two kinds of information that exhibited differences in their memorability. Moreover, because storage and retrieval conditions were held constant for the novel and familiar words, the difference in their memorability can be attributed to differences in their encoding.

As mentioned earlier, we selected the seed voxels for our analysis in the hippocampus because of considerable evidence in the literature about the involvement of the hippocampus in novelty detection [1,2,5-20,47]. It is also generally accepted that novelty detection involves regions beyond the hippocampus $[1,2,12,17,19,20]$. The networks identified in our study also included regions from widely distributed cortical and subcortical areas. Thus, the present results indicate that the neurophysiological response to novelty and familiarity is associated with the coherent activity of networks of spatially remote cortical and subcortical regions in which the hippocampus plays a prominent role.

Perhaps the most intriguing specific finding that emerged from our study is that the same region of the hippocampus (same seed voxels) participated in two different networks depending on whether subjects were engaged in encoding novel or familiar words. This finding provides support for the concept of neural context, the principle that the neurocognitive function served by a specific brain region in a given task is determined through its interaction with other brain regions $[40,41,48]$. Put differently, a single brain region may underlie different cognitive processes depending on which other brain regions are co-activated with it-its neural context. In the present study, the anterior ventral hippocampal seed region participated in two different non-overlapping networks, one recruited when subjects encoded novel words and the other when subjects encoded familiar words. The only region common to both networks was the hippocampal seed region, supporting the notion that the function of this region (novelty or familiarity discrimination) changes depending on its neural context.

In the present experiment, brain activity was measured when words were presented for the first time and again when words were repeated. Similar paradigms have been used to examine the neural mechanisms underlying repetition priming [49-56] (for reviews, see [4]). In these studies, brain activity for novel unprimed stimuli has typically been greater, especially in the left prefrontal cortex, than brain activity for familiar primed stimuli. This difference in activity, termed neural priming, has been hypothesized to underlie behavioral repetition priming. However, an equally plausible interpretation of these findings is put forth by the novelty encoding hypothesis: activation in left prefrontal cortex is greater for novel unprimed stimuli than familiar primed stimuli because the former type, by virtue of its novelty, is more likely to be encoded into long-term memory. At present, these two interpretation cannot be disentangled (for a discussion of this issue, see [57]). The present results could therefore be interpreted as networks underlying primed and unprimed items.

At the present early stage of the search for evidence of coherent neural networks of any kind, including those involved in novelty and familiarity processing, it would be unwise to take the data provided by any single study too seriously. Because there have been no comparable studies reported in the literature, describing interregional correlations associated with novelty discrimination, it is not possible for us to compare our findings to what has already been published. It is not without interest, however, that the seed voxels in the left MTL that we have just discussed at some length turned out to be within a few millimeters from a voxel that Constable et al. [7] identified as a region that was more active when 
subjects viewed complex novel scenes rather than complex familiar scenes.

Additionally, it is also important to worry about the possibility of "identifying" collections of brain regions that satisfy the definition of a network, but which in fact have come about purely by chance. This kind of concern is always present in all studies involving some 60,000 voxels of the brain model that is commonly used [58]. We did take what we thought were appropriate steps to guard against false positives (limited search space, permutation test, bootstrapping), but there is no guarantee that we have not included some brain regions in our networks that really do not belong there.

In the present study, the manipulation of stimulus history, and hence novelty and familiarity, was orthogonal to the other independent variables. The aim of such a design was to remove the confound between novelty and familiarity on the one hand, and task outcome (new and old during recognition) on the other. Nonetheless, it is possible that subjects became aware of the nature of each study list (novel or familiar) and, therefore, the differences that we have observed and interpreted as differences in the neural response to novelty and familiarity, at least partly, are attributable to the phenomenal awareness of the contents of the list. The present experiment did not address this issue and we leave this as a problem for future studies.

In summary, the findings we have reported here have indicated that large-scale coherent functional networks are associated with discrimination of novel and familiar information, even under conditions in which the novelty or familiarity of the items is irrelevant to the focal task in which the subjects are engaged. Our findings illustrate the possibility that individual networks operate coherently when the task involves novel information but not familiar, whereas others may do so when the task involves familiar but not novel information. Furthermore, they illustrate the participation of the hippocampus in both types of network, its function determined by its neural context. Our present findings, of course, in no way preclude the possibility of the existence of other comparable networks involving other regions of the brain. Finally, the study we have reported here can be seen as contributing to the growing consensus that cognitive processes are mediated by large-scale neural networks $[25,28,33,42,43,45,46,48]$, and to the appreciation of the usefulness of covariance-based analyses $[44,59]$.

\section{Acknowledgements}

We would like to thank D. Hussey, K. Cheung, and the staff at the PET centre at the Clarke Institute of Psychiatry for their help in data collection. We would also like to thank Holly Cormier for assistance with the creation of the auditory stimuli. This research was supported by a foundation of Anne and Max Tanenbaum in support of research in cognitive neuroscience, and by the Natural Sciences and
Engineering Research Council of Canada (Grant 8632). $\mathrm{RH}$ is supported by a McDonnell foundation investigator initiated grant to ARM and a Tanenbaum postdoctoral fellowship.

\section{References}

[1] Friedman D, Cycowicz YM, Gaeta H. The novelty P3: an event-related brain potential (ERP) sign of the brain's evaluation of novelty. Neuroscience and Biobehavioral Reviews 2001;25(4):35573.

[2] Knight RT, Nakada T. Cortico-limbic circuits and novelty: a review of EEG and blood flow data. Reviews in the Neurosciences 1998;9(1):57-70

[3] Sokolov EN. Higher nervous functions: the orienting reflex. Annual Review of Physiology 1963;25:545-80.

[4] Wiggs CL, Martin A. Properties and mechanisms of perceptual priming. Current Opinion in Neurobiology 1998;8(2):227-33.

[5] Aggleton JP, Brown MW. Episodic memory, amnesia, and the hippocampal-anterior thalamic axis. Behavioral and Brain Sciences 1999;22:425-89.

[6] Brown MW, Aggleton JP. Recognition memory: what are the roles of the perirhinal cortex and hippocampus? Nature Reviews Neuroscience 2001;2:51-61.

[7] Constable RT, Carpentier A, Pugh K, Westerveld M, Oszunar Y, Spencer DD. Investigation of the human hippocampal formation using a randomized event-related paradigm and $z$-shimmed functional MRI. NeuroImage 2000;12(1):55-62.

[8] Dolan RJ, Fletcher PC. Dissociating prefrontal and hippocampal function in episodic memory encoding. Nature 1997;388:582-5.

[9] Gabrieli JDE, Brewer JB, Desmond JE, Glover GH. Separate neural bases of two fundamental memory processes in the human medial temporal lobe. Science 1997;276(5310):264-6.

[10] Grunwald T, Lehnertz K, Heinze H-J, Helmstaedter C, Elger CE. Verbal novelty detection within the human hippocampus proper. Proceedings of the National Academy of Science of the United States of America 1998;95(6):3193-7.

[11] Habib R, Lepage M. Novelty assessment in the brain. In: Tulving E, editor. Memory, consciousness, and the brain: The Tallinn Conference. Philadelphia: Psychology Press; 2000. p. 265-77.

[12] Kirchhoff BA, Wagner AD, Maril A, Stern CE. Prefrontal-temporal circuitry for episodic encoding and subsequent memory. Journal of Neuroscience 2000;20(16):6173-80.

[13] Knight RT. Contribution of human hippocampal region to novelty detection. Nature 1996;383(6597):256-9.

[14] Manns JR, Stark CE, Squire LR. The visual paired-comparison task as a measure of declarative memory. Proceedings of the National Academy of Science of the United States of America 2000;97(22):12375-9.

[15] Martin A, Wiggs CL, Weisberg J. Modulation of human medial temporal lobe activity by form, meaning, and experience. Hippocampus 1997;7:587-93.

[16] McKee RD, Squire LR. On the development of declarative memory. Journal of Experimental Psychology: Learning, Memory, and Cognition 1993;19(2):397-404.

[17] Saykin AJ, Johnson SC, Flashman LA, McAllister TW, Sparling M, Darcey TM, et al. Functional differentiation of medial temporal and frontal regions involved in processing novel and familiar words: an fMRI study. Brain 1999;122(10):1963-71.

[18] Stern CE, Corkin S, Gonzalez RG, Guimaraes AR, Baker JR, Jennings PJ, et al. The hippocampal formation participates in novel picture encoding: evidence from functional magnetic resonance imaging. Proceedings of the National Academy of Science of the United States of America 1996;93:8660-5. 
[19] Tulving E, Markowitsch HJ, Craik FIM, Habib R, Houle S. Novelty and familiarity activations in PET studies of memory encoding and retrieval. Cerebral Cortex 1996;6:71-9.

[20] Tulving E, Markowitsch HJ, Kapur S, Habib R, Houle S. Novelty encoding networks in the human brain: positron emission tomography data. NeuroReport 1994;5:2525-8.

[21] Kormi-Nouri R, Nilsson L-G, Ohta N. The novelty effect: support for novelty/encoding hypothesis. International Journal of Psychology 2000;35(3-4):196-7.

[22] Tulving E, Kroll N. Novelty assessment in the brain and long-term memory encoding. Psychonomic Bulletin and Review 1995;2(3):38790.

[23] Kinsbourne M, George J. The mechanism of the word-frequency effect on recognition memory. Journal of Verbal Learning and Verbal Behavior 1974;13(1):63-9.

[24] Düzel E, Cabeza R, Picton TW, Yonelinas AP, Scheich H, Heinze $\mathrm{H}-\mathrm{J}$, et al. Task-related and item-related brain processes of memory retrieval. Proceedings of the National Academy of Science of the United States of America 1999;96(4):1794-9.

[25] Della-Maggiore V, Sekuler AB, Grady CL, Bennett PJ, Sekuler $\mathrm{R}$, McIntosh AR. Corticolimbic interactions associated with performance on a short-term memory task are modified by age. Journal of Neuroscience 2000;20(22):8410-6.

[26] McIntosh AR, Nyberg L, Bookstein FL, Tulving E. Differential functional connectivity of prefrontal and medial temporal cortices during episodic memory retrieval. Human Brain Mapping 1997;5:323-7.

[27] McIntosh AR, Rajah MN, Lobaugh NJ. Interactions of prefrontal cortex in relation to awareness in sensory learning. Science 1999;284(5419):1531-3.

[28] Nyberg L, Persson J, Habib R, Tulving E, McIntosh AR, Cabeza $R$, et al. Large scale neurocognitive networks underlying episodic memory. Journal of Cognitive Neuroscience 2000;12:16373.

[29] Craik FIM, Lockhart RS. Levels of processing: a framework for memory research. Journal of Verbal Learning and Verbal Behavior 1972;11:671-84.

[30] Craik FIM, Tulving E. Depth of processing and the retention of words in episodic memory. Journal of Experimental Psychology: General 1975;104:268-94.

[31] Kapur S, Craik FIM, Tulving E, Wilson AA, Houle S, Brown GM. Neuroanatomical correlates of encoding in episodic memory: levels of processing effect. Proceedings of the National Academy of Science of the United States of America 1994;91(6):2008-11.

[32] Talairach J, Tournoux P. Co-planar stereotaxic atlas of the human brain. New York: Thieme Medial Publishers Inc.; 1988.

[33] Friston KJ. Functional and effective connectivity in neuroimaging: a synthesis. Human Brain Mapping 1994;2:56-78.

[34] Milan L, Whittaker J. Application of the parametric bootstrap to models that incorporation a singular value decomposition. Royal Statistical Society Journal, Series C: Applied Statistics 1995;44:3149.

[35] Lepage M, Habib R, Tulving E. Hippocampal PET activations of memory encoding and retrieval: the HIPER model. Hippocampus 1998;8(4):312-22.

[36] Schacter DL, Wagner AD. Medial temporal lobe activations in fMRI and PET studies of episodic encoding and retrieval. Hippocampus 1999;9(1):7-24.

[37] Lancaster JL, Rainey LH, Summerlin JL, Freitas CS, Fox PT, Evans AC, et al. Automated labeling of the human brain: a preliminary report on the development and evaluation of a forward-transform method. Human Brain Mapping 1997;5(4):238-42.

[38] Lancaster JL, Woldorff MG, Parsons LM, Liotti M, Freitas CS, Rainey L, et al. Automated Talairach atlas labels for functional brain mapping. Human Brain Mapping 2000;10(3):120-31.
[39] McIntosh AR, Grady CL, Haxby JV, Ungerleider LG, Horwitz B. Changes in limbic and prefrontal functional interactions in a working memory task for faces. Cerebral Cortex 1996;6:571-84.

[40] McIntosh AR. Understanding neural interactions in learning and memory using functional neuroimaging. Annals of the New York Academy of Sciences 1998;30(855):556-71

[41] McIntosh AR. Mapping cognition to the brain through neural interactions. Memory 1999;7(5-6):523-48.

[42] Bressler SL. Large-scale cortical networks and cognition. Brain Research Reviews 1995;20:288-304.

[43] Büchel C, Friston KJ. Modulation of connectivity in visual pathways by attention: cortical interactions evaluated with structural equation modelling and fMRI. Cerebral Cortex 1997;7(8):768-78.

[44] McIntosh AR, Gonzalez-Lima F. Structural equation modeling and its application to network analysis in functional brain imaging. Human Brain Mapping 1994;2(1-2):2-22.

[45] Mesulam MM. From sensation to cognition. Brain 1998;121:101352.

[46] Nyberg L, McIntosh AR, Cabeza R, Nilsson L-G, Houle S, Habib $\mathrm{R}$, et al. Network analysis of positron emission tomography regional cerebral blood flow data: ensemble inhibition during episodic memory retrieval. Journal of Neuroscience 1996;16(11):3753-9.

[47] Knight RT, Scabini D. Anatomic bases of event-related potentials and their relationship to novelty detection in humans. Journal of Clinical Neurophysiology 1998;15(1):3-13.

[48] McIntosh AR. Toward a network theory of cognition. Neural Networks 2000;13(8-9):861-70.

[49] Buckner RL, Goodman J, Burock M, Rotte M, Koutstaal W, Schacter D, et al. Functional-anatomic correlates of object priming in humans revealed by rapid presentation event-related fMRI. Neuron 1998;20:285-96.

[50] Buckner RL, Koutstaal W. Functional neuroimaging studies of encoding, priming, and explicit memory retrieval. Proceedings of the National Academy of Science of the United States of America 1998;95:891-8.

[51] Buckner RL, Koutstaal W, Schacter DL, Rosen BR. Functional MRI evidence for a role of frontal and inferior temporal cortex in amodal components of priming. Brain 2000;123:620-40.

[52] Demb JB, Desmond JE, Wagner AD, Vaidya CJ, Glover GH, Gabrieli JDE. Semantic encoding and retrieval in the left inferior prefrontal cortex: a functional MRI study of task difficulty and process specificity. Journal of Neuroscience 1995;15(9):5870-8.

[53] Koutstaal W, Wagner AD, Rotte M, Maril A, Buckner RL, Schacter DL. Perceptual specificity in visual object priming: fMRI evidence for a laterality difference in fusiform cortex. Neuropsychologia 2001;39(2):184-99.

[54] Wagner AD, Desmond JE, Demb JB, Glover GH, Gabrieli JDE. Semantic repetition priming for verbal and pictorial knowledge: a functional MRI study of left inferior prefrontal cortex. Journal of Cognitive Neuroscience 1997;9(6):714-26.

[55] Wagner AD, Koutstaal W, Maril A, Schacter DL, Buckner RL. Task-specific repetition priming in left inferior prefrontal cortex. Cerebral Cortex 2000;10(12):1176-84.

[56] Wagner AD, Maril A, Schacter DL. Interactions between forms of memory: when priming hinders new episodic learning. Journal of Cognitive Neuroscience Suppl 2;12:52-60.

[57] Habib R. On the relation between conceptual priming, neural priming, and novelty assessment. Scandinavian Journal of Psychology 2001;42(3):187-95.

[58] Tulving E, Habib R, Nyberg L, Lepage M, McIntosh AR. Positron emission tomography correlations in and beyond medial temporal lobes. Hippocampus 1999;9(1):71-82.

[59] McIntosh AR, Bookstein FL, Haxby JV, Grady CL. Spatial pattern analysis of functional brain images using partial least squares. NeuroImage 1996;3:143-57. 